H I G H L I G H T S

\title{
Regulating olfactory receptors
}

Two new studies provide evidence that the monoallelic expression pattern of olfactory receptors (ORs) in mammals is regulated by a negative feedback mechanism.

The exquisite sensitivity of the mammalian olfactory system depends on functionally distinct neuronal populations that project to precisely defined glomeruli in the olfactory bulb (see figure). Establishment of these discrete populations is a function of the 'one neuron-one receptor' rule. This rule describes how each olfactory neuron expresses only one allele of one OR gene from the more than 1,000 in the mouse genome. In an effort to enhance our understanding of how this process is regulated, two research groups tested the hypothesis that the product of an expressed OR gene prevents activation of its counterparts.

Sakano and colleagues used transgenic constructs in yeast artificial chromosomes to manipulate expression of the MOR2 8 gene in mice. Transformation of mice with enhanced green fluorescent protein (EGFP)-tagged MOR28 from which the entire coding sequence had been deleted resulted in co-expression of EGFP and endogenous MOR28 in many cells. Other EGFP-positive neurons of the olfactory epithelium expressed different endogenous OR genes, indicating that a product of the MOR2 8 coding sequence is required to prevent the expression of other ORs.

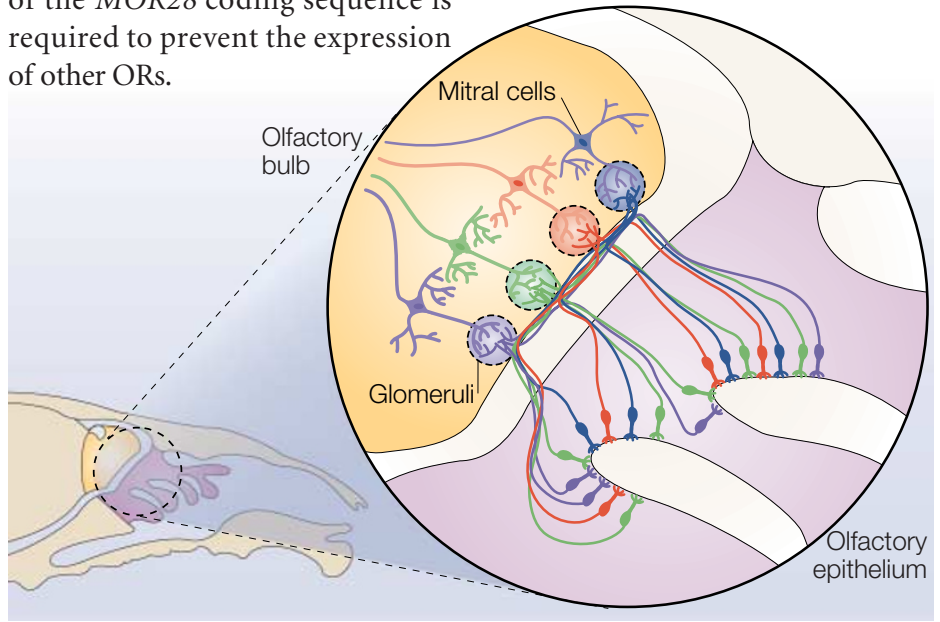

Lewcock and Reed extended these findings by showing that it is the protein product of OR genes that activates the negative regulatory loop. To distinguish between possible transcriptional and translational activation of inhibition, the authors used an OR-promoter-driven transgene in which the coding sequence was present but untranslatable. The presence of this transgene did not prevent the expression of other OR alleles - strong evidence that it is OR protein rather than messenger RNA that directs the selective expression of a single OR allele.

Sakano et al. also identified a cis-acting regulatory element upstream of the MOR28 gene cluster that is necessary for gene expression. These authors suggest a model in which a transcription-activating complex is formed in this regulatory region. This complex would then interact with the promoter of just one gene in the cluster, expression of which would feed back to prevent the induction of other OR genes.

Suzanne Farley

\section{(2) References and links} ORIGINAL RESEARCH PAPERS Serizawa, S. et al. Negative feedback regulation ensures the one receptor-one olfactory neuron rule in mouse. Science 302, 2088-2094 (2003) | Lewcock, J. W. \& Reed, R. R. A feedback mechanism regulates monoallelic odorant receptor expression. Proc. Natl Acad. Sci. USA 101, 1069-1074 (2004) FURTHER READING Laurent, G. Olfactory network dynamics and the coding of multidimensional signals. Nature Rev. Neurosci. 3, 884-895 (2002)

\section{IN BRIEF}

\section{COGNITIVE NEUROSCIENCE}

Shrinkage of the entorhinal cortex over five years predicts memory performance in healthy adults.

Rodrigue, K. M. \& Raz, N. J. Neurosci. 24, 956-963 (2004)

In a five-year longitudinal study, Rodrigue and Raz tested whether shrinkage of the entorhinal cortex, hippocampus or prefrontal cortex of healthy adults could predict episodic memory performance. Although none of the regional volumes was associated with memory performance, greater shrinkage in the entorhinal cortex was associated with poorer memory performance at the end of the study. The shrinkage of the hippocampus and prefrontal cortex did not predict memory performance.

\section{SENSORY PHYSIOLOGY}

\section{Mustard oils and cannabinoids excite sensory nerve} fibres through the TRP channel ANKTM1.

Jordt, S.-E. et al. Nature 427, 260-265 (2004)

Isothiocyanate compounds, such as the active ingredients in mustard, produce pain, inflammation and hypersensitivity when applied to the skin. Jordt and colleagues show that the application of mustard oil depolarizes the same primary sensory neurons as are activated by capsaicin, the 'hot' ingredient in chilli peppers, and by $\Delta^{9}$-tetrahydrocannabinol (THC), the active ingredient in marijuana. Both allyl isothiocyanate and THC activate a TRP (transient receptor potential) ion channel called ANKTM1.

\section{DEVELOPMENT}

\section{Paraxial mesoderm specifies zebrafish primary} motoneuron subtype identity.

Lewis, K. E. \& Eisen, J. S. Development 131, 891-902 (2004)

Signals from the paraxial mesoderm specify distinct populations of motor neurons in vertebrates. Lewis and Eisen show that the paraxial mesoderm is also the origin of signals that control the more precise patterning of primary motor neurons in the zebrafish, giving rise to a segmentally repeated pattern of two subtypes of motor neuron. In the absence of paraxial mesoderm-derived signals, primary motor neurons seem to have a hybrid identity.

\section{GLIA}

\section{Astrocyte-mediated activation of neuronal kainate receptors.}

Liu, Q.-S. et al. Proc. Natl Acad. Sci. USA 6 February 2004 (10.1073/pnas.0306731101)

To test whether glutamate released by astrocytes in rat hippocampal slices activates kainate receptors on neighbouring neurons, Liu et al. used $o$-nitrophenyl-EGTA to uncage $\mathrm{Ca}^{2+}$. Increases in intracellular $\mathrm{Ca}^{2+}$ in astrocytes increased action-potential-driven spontaneous inhibitory postsynaptic currents in nearby interneurons. The effect was blocked by kainate receptor antagonists but not by antagonists of other glutamate receptor subtypes. Astrocytes might therefore be an important modulator of neuronal function in the hippocampus. 